\title{
Electromagnetic Shower Simulation for CORSIKA 8
}

\section{Jean-Marco Alameddine, ${ }^{a, *}$ Jaime Alvarez-Muñiz, ${ }^{b}$ Juan Ammerman-Yebra, ${ }^{b}$ Lars Bollmann, ${ }^{a}$ Wolfgang Rhode, ${ }^{a}$ Maximilian Sackel, ${ }^{a}$ Alexander Sandrock, ${ }^{c}$ Jan Soedingrekso ${ }^{a}$ and Enrique $\mathrm{Zas}^{b}$ on behalf of the CORSIKA 8 Collaboration} (a complete list of authors can be found at the end of the proceedings)

${ }^{a}$ Technical University Dortmund, Otto-Hahn-Str. 4a, 44227 Dortmund, Germany

${ }^{b}$ Instituto Galego de Física de Altas Enerxías (IGFAE),

Universidade de Santiago de Compostela, 15782 Santiago de Compostela, Spain

${ }^{c}$ National Research Nuclear University MEPhI (Moscow Engineering Physics Institute), Kashirskoe shosse 31, 115409 Moscow, Russia

E-mail: jean-marco.alameddine@udo.edu

Extensive air showers in astroparticle physics experiments are commonly simulated using CORSIKA. The electromagnetic shower component has been treated using EGS4 in the Fortran 77based versions, which have been developed in the last thirty years. Currently, CORSIKA is being restructured and rewritten in $\mathrm{C}++$, leading to the new version CORSIKA 8. In this process, the electromagnetic component is now being treated by the high-energy lepton and photon propagator PROPOSAL. Originally designed for the efficient simulation of high-energy muons and tau-leptons in large volume neutrino telescopes, the Monte Carlo library PROPOSAL has been extended to also treat electrons, positrons, and high-energy photons. Validating this new implementation of the electromagnetic shower model is very important. In this talk, the electromagnetic shower component simulated with PROPOSAL is compared to previous versions of CORSIKA, the air shower simulator AIRES as well as the electromagnetic shower tool ZHS, which is optimized for the radio signal. This includes comparisons of the underlying theoretical models as well as lateral and longitudinal shower characteristics, especially of parameters relevant for the radio component such as the charge excess.

$37^{\text {th }}$ International Cosmic Ray Conference (ICRC 2021)

July 12th - 23rd, 2021

Online - Berlin, Germany

\footnotetext{
${ }^{*}$ Presenter
} 


\section{Introduction}

For decades, many astroparticle experiments have simulated extensive air showers using CORSIKA [1]. In recent years, a major effort has been started to rewrite CORSIKA in modern C++, resulting in the new version CORSIKA 8. In the course of this undertaking, the propagation of electromagnetic particles has been transferred from a modified version of EGS4 [2] to the PROPOSAL library [3-5]. This contribution is dedicated to the systematic comparison of electromagnetic showers simulated in the current version of CORSIKA 8 to established frameworks, including CORSIKA 7, the air shower simulation program AIRES [6] and the electromagnetic cascade simulation code ZHS MC [7]. In the past, AIRES and ZHS MC have already been compared against GEANT4 and have been found to be in good agreement for simulations in homogeneous ice [8].

This proceeding is divided in two main parts. In Section 2 we compare the theoretical description of the electromagnetic interaction processes in the different codes. Section 3 describes the results of our simulations and compares the longitudinal and lateral shower development as well as the track lengths of simulated showers.

\section{Comparison of theoretical descriptions of EM processes}

The comparison of the theoretical models used in the different shower frameworks includes energy loss cross sections as well as the scattering models. A discussion is presented of the different treatments of energy thresholds to remove low energetic particles and of cuts to decide whether interactions are treated stochastically or as contributing to the continuous energy loss.

In all frameworks but AIRES, the electromagnetic model is similar to the Electron Gamma Shower code system EGS4 [2]. While in ZHS MC and PROPOSAL, the models are mainly based on EGS4, CORSIKA 7 uses EGS4 directly in a modified version. For AIRES, electromagnetic processes are based on several parametrizations different from EGS, which will be described in more detail later in this chapter. Since EGS4 is a built-in system producing the showers with limited access for external frameworks, the lepton propagator PROPOSAL is being used in CORSIKA 8 as an external library. Thereby, PROPOSAL provides the physical description of the electromagnetic shower component, while the overall task of the shower generation remains in the CORSIKA framework.

The two dominating processes in an electromagnetic shower are electron-positron pair production by photons and bremsstrahlung losses of electrons and positrons. In CORSIKA 7, the bremsstrahlung parametrization of Koch \& Motz [9] is used for energies above $50 \mathrm{MeV}$, while for lower energies, tabulated empirical corrections are applied. The same bremsstrahlung parametrization has been implemented in PROPOSAL. In ZHS MC, the parametrization of Stanev \& Vankov [10] is used, which is based on [11, 12], with corrections at low energies from [9]. The framework AIRES uses a cross section based on a parametrization by Rossi \& Greisen [13]. In all frameworks, the LPM effect is included, although it can currently not be used in CORSIKA 8.

The same formalism to parametrize the bremsstrahlung cross section is also used for the pair production cross section, except for CORSIKA 8, where a parametrization by Tsai is used [14]. Using two different parametrizations to describe the bremsstrahlung and pair production processes 
in CORSIKA 8 is not entirely consistent, however, interchanging the pair production cross section resulted in negligible differences compared to other uncertainties.

Regarding ionization and the production of knock-on electrons, CORSIKA 7, CORSIKA 8 and ZHS MC all use the Berger \& Seltzer parametrization [15] of the Bhabha and Møller scattering for electrons and positrons, respectively. In AIRES, a fit to GEANT3 calculations with a distinction between continuous and discrete losses at an energy of $1 \mathrm{MeV}$ is made. For a better comparison with the other frameworks, its continuous energy loss has been substituted by that of Berger \& Seltzer [15]. All frameworks also include density correction effects.

Further interactions implemented in all frameworks are the annihilation of positrons with atomic electrons and Compton scattering of photons. Together with Møller and Bhabha scattering, they are relevant for the charge excess of an air shower, and therefore the radio signal [7].

Only in CORSIKA 7 and AIRES, the photohadronic and the photoelectric effects are implemented. For this work, the photohadronic interaction has been deactivated in AIRES to compare only the purely electromagnetic component of the showers. In CORSIKA 7, the photoelectric effect also includes the fluorescence loss. Further processes that are only taken into account by CORSIKA 7 are coherent Rayleigh scattering and the production of muon pairs induced by photons.

In PROPOSAL, the production of electron-positron pairs induced by leptons colliding with nuclei as well as inelastic nuclear interactions are also implemented. The latter, however, is only included as an energy loss process, but not yet as a source of hadronic secondary particles.

For the description of multiple scattering, the Highland approximation [16] of Molière theory is used in all frameworks. The deflection of particles in stochastic interactions is included in CORSIKA 7. These effects are also available in PROPOSAL, but not yet implemented in the interface to CORSIKA 8. In AIRES, Coulomb scattering is implemented as a deflection process.

Besides the physical models, the implementation and treatment of energy cuts are important to compare the simulations. The cuts can be divided into a particle energy cut below which particles are not further propagated. Furthermore, there are energy loss cuts defining the energy above which losses are treated stochastically, while below the cut, they are treated as a continuous energy loss between two interactions.

In AIRES, the user can define particle cuts which need to be above a kinetic energy of $80 \mathrm{keV}$ for the particles in an electromagnetic shower. Energy loss cuts are set in the code and can not be changed externally. For this work, the AIRES code has been modified in such a way that the energy loss cuts match those used in the other frameworks. In ZHS MC, energy cuts are carefully linked to the cut between discrete and continuous losses, without an option to change them individually. For comparison reasons, an independent particle cut has been introduced in an attempt to match the cross sections and the same integration limits of the other frameworks. Since the particle cuts in AIRES and ZHS MC have been modified from their initial values, these routines may not perform with their optimal accuracy.

In CORSIKA 7, the particle cuts can be changed as long as they are above $10 \mathrm{keV}$ for electrons and positrons, and above $1 \mathrm{keV}$ for photons. The energy loss cut can not be set in CORSIKA 7. In CORSIKA 8, both the energy loss cut and the particle cut can be adjusted independently for the different particle types. For comparison reasons, all particle cuts have been set to $4 \mathrm{MeV}$ and energy loss cuts to half of the particle cut since CORSIKA 8 is known to produce stable results for these settings. 

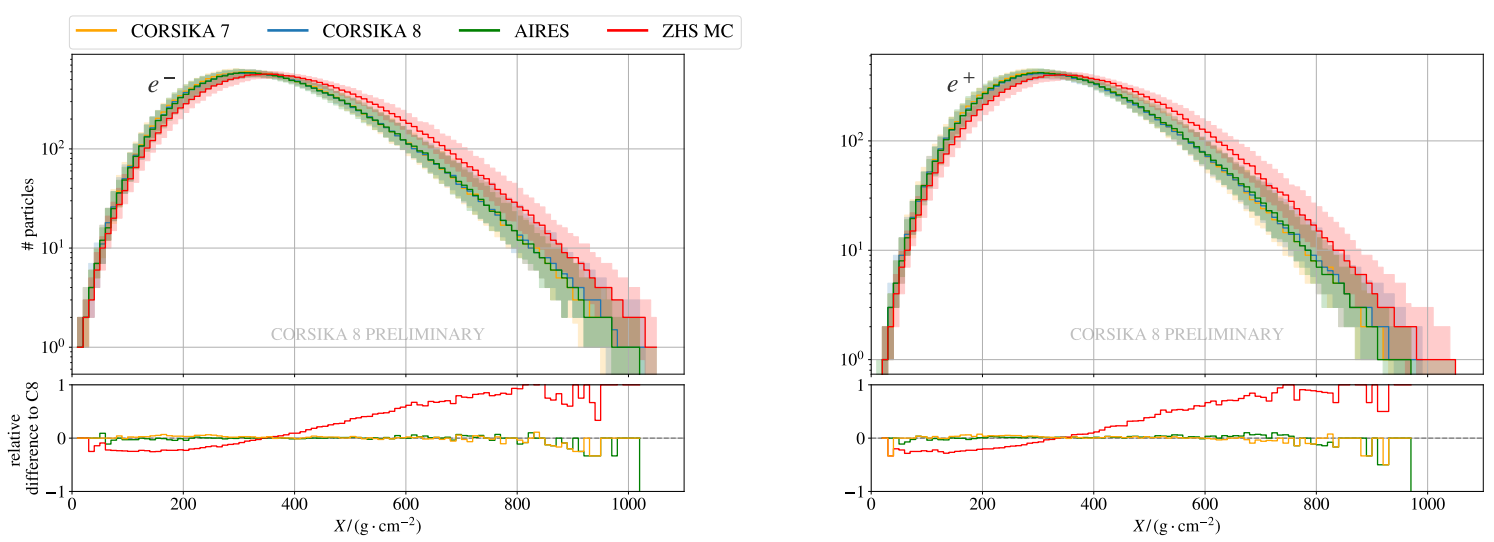

Figure 1: Longitudinal profile for 200 showers initiated by $1 \mathrm{TeV}$ electrons. The relative difference to CORSIKA 8 is defined as $\frac{m-m_{\mathrm{C} 8}}{m_{\mathrm{C} 8}}$ with the median $m$ of the particle number.

\section{Comparison of simulated shower parameters}

To validate the current status of the simulations of the electromagnetic shower component in CORSIKA 8 using PROPOSAL, relevant shower observables are calculated and compared to the results from simulations created with other shower simulation codes. These comparisons are vital to understand the current status of CORSIKA 8, but also to highlight the remaining limitations when interpreting the physical results which are obtained with it.

For the results presented in this contribution, we used version 7.7410 of CORSIKA 7 [1], version 19.04.00 of AIRES [6], the release tagged icrc-2021 for CORSIKA 8 [17] and the most recent version of ZHS MC [18]. All the comparisons that are presented have been obtained by simulating an electromagnetic shower induced by an electron with an initial energy of $1 \mathrm{TeV}$. The particle threshold has been set to $4 \mathrm{MeV}$, i.e. shower particles with a kinetic energy below this threshold are discarded. The energy loss cuts have been set to $2 \mathrm{MeV}$ for photons and to $2.255 \mathrm{MeV}$ for electrons and positrons.

For CORSIKA 7, all simulations have been made using the U.S. Standard Atmosphere model, an inhomogeneous density profile of the atmosphere. For ZHS MC, all air showers were simulated in a homogeneous atmosphere, as this is the only option available, with a density of $1200 \mathrm{~g} \mathrm{~m}^{-3}$. CORSIKA 8 and AIRES simulations were run for both inhomogeneous and homogeneous atmospheres. Since there are no particles decaying in purely electromagnetic showers, we do not expect this to significantly affect the comparisons of the longitudinal shower development in terms of grammage.

\subsection{Longitudinal shower development}

The longitudinal shower development is analyzed by counting the total number of particles crossing planes along the shower axis and perpendicular to it. This is shown for the different particle types in Figure 1, where the longitudinal profile has been calculated using the simulations of 200 showers. Here, the solid lines indicate the median, while the shaded bands display the interquartile range of the particle number. 


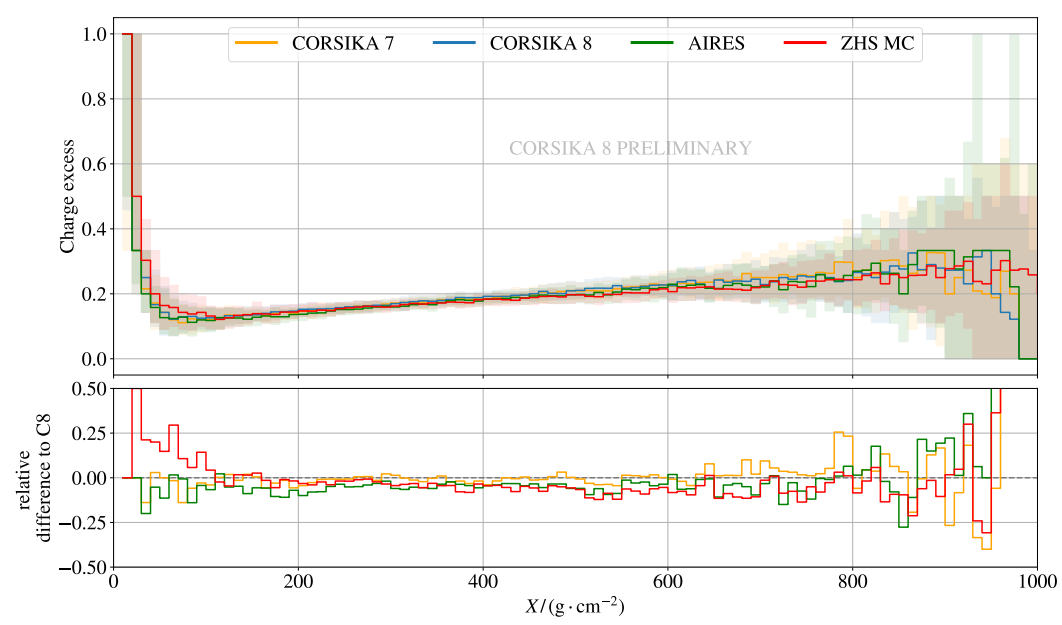

Figure 2: Longitudinal distribution of the charge excess, defined as $\frac{N_{e^{-}}-N_{e^{+}}}{N_{e^{-}+N_{e^{+}}}}$, for 200 showers initiated by $1 \mathrm{TeV}$ electrons.

While the general shapes of the longitudinal shower distribution agree, a displacement of the showers simulated with ZHS MC towards larger depths is clearly visible. For CORSIKA 7 , CORSIKA 8 and AIRES, the medians of the distribution all agree within $5 \%$.

Figure 2 shows the longitudinal development of the difference between the electron and positron number, called charge excess. This effect is caused by the ionization of atomic electrons either by charged leptons or by Compton scattering as well as by annihilation of positrons. The resulting charge excess is, together with the geomagnetic contribution, a mechanism for radio emission in air showers, and shows an agreement between the different frameworks that is within $10 \%$ around the depth of the shower maximum.

\subsection{Lateral shower development}

The lateral shower development, i.e. the distribution of the shower perpendicular to the shower axis, is computed by selecting all particles passing a given observation level and calculating their distance to the shower axis. The resulting lateral particle distribution of electrons and positrons is shown for simulations in an inhomogeneous atmosphere in Figure 3a, and for simulations in a homogeneous atmosphere in Figure 3b. For the inhomogeneous medium, the observation level has been set to $8600 \mathrm{~m}$ above sea level, which corresponds to an atmospheric depth of approximately $335 \mathrm{~g} \mathrm{~cm}^{-3}$, while for the homogeneous medium, the observation level has been set directly to the shower maximum. The median number of particles is depicted by the solid lines, the shaded bands indicate the interquartile range of the particle number.

The comparisons show that in both the inhomogeneous and homogeneous atmosphere, the lateral profiles from the CORSIKA 8 simulations are shifted slightly closer to the shower axis compared to the simulations done with the other frameworks. This indicates that showers produced by CORSIKA 8 have a smaller lateral spread, which can be understood since not all contributing processes have yet been implemented in CORSIKA 8. For example, bremsstrahlung photons produced by electrons and positrons inherit the direction of the initial lepton, neglecting the photon emission angle. 

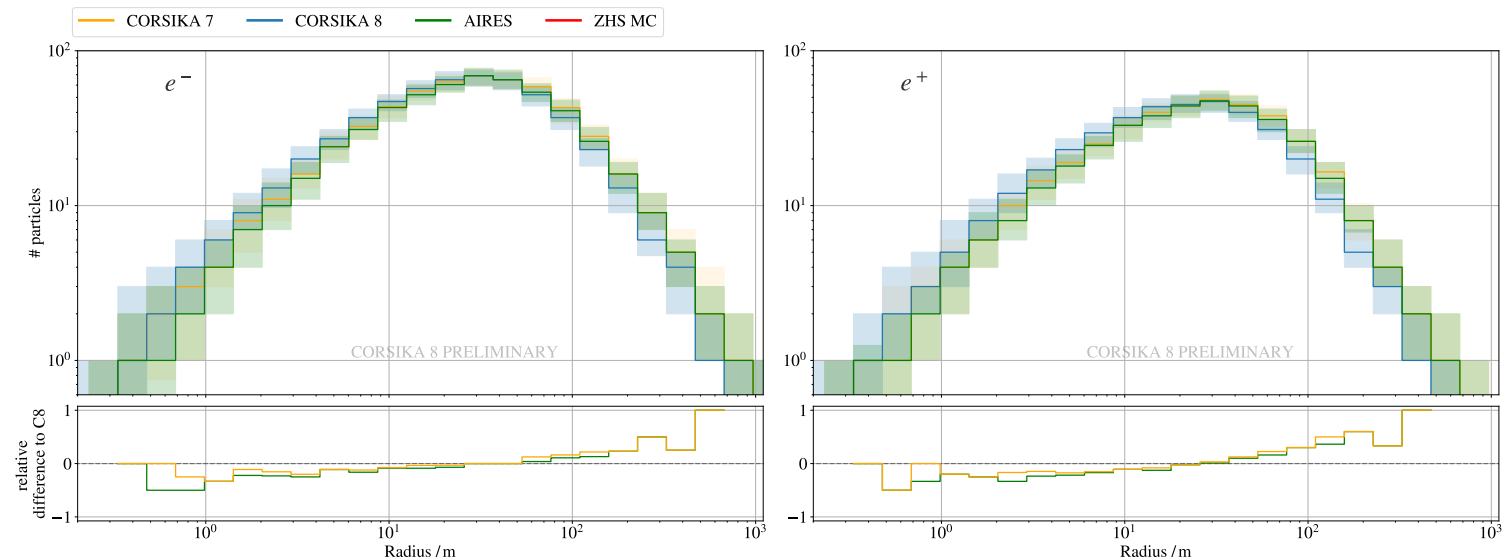

(a) Distributions for an observation height of $8600 \mathrm{~m}$ in an inhomogeneous atmosphere.
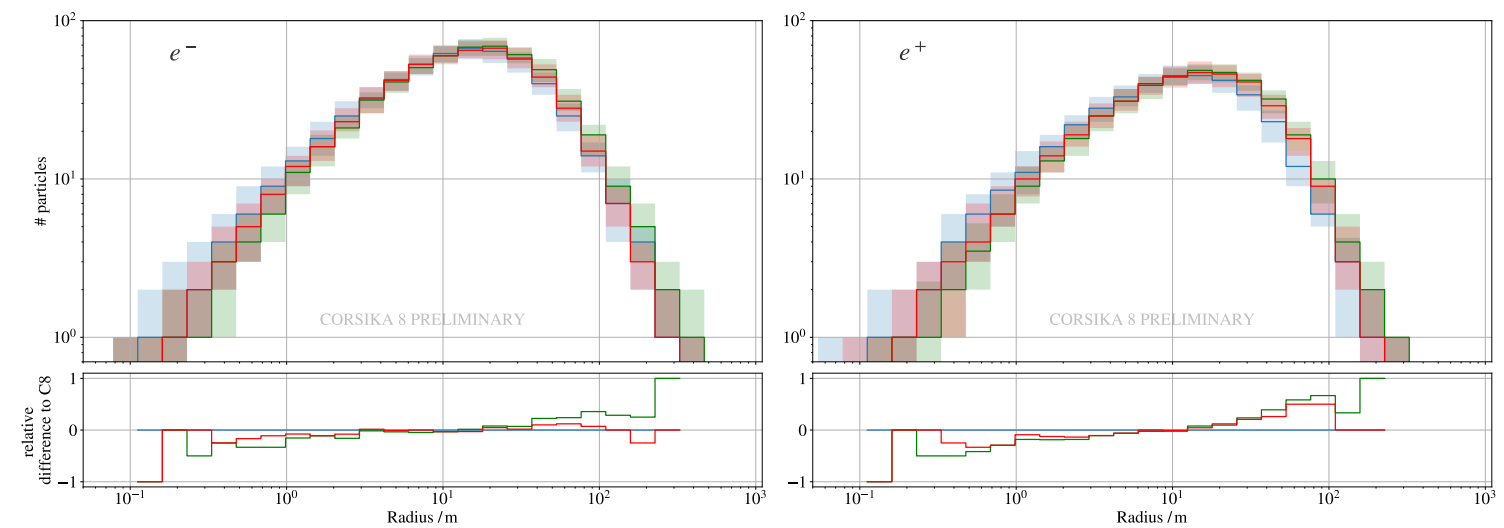

(b) Distributions for an observation height at the shower maximum in a homogeneous atmosphere.

Figure 3: Lateral particle distributions for 200 showers initiated by $1 \mathrm{TeV}$ electrons.

\subsection{Shower track lengths}

For our purposes, the track length is defined as the sum of the lengths of all electron and positron tracks, with the projected track length defined as the sum of all tracks projected onto the shower axis. Both quantities are calculated for 200 showers in both homogeneous and inhomogeneous atmospheres, simulated with the different frameworks.

In addition, the excess track length is calculated, which is the sum of all electron track lengths in the shower minus the sum of all positron track lengths, while the projected excess track length is defined analogously to the projected charged track length. The projected excess track length is an important quantity in simulating the radio emission of air showers, as it is the quantity that establishes the normalization of the electric field spectrum [7]. The distributions of the projected excess track lengths are shown in Figure 4, the medians and standard deviations of all track length observables are presented in Table 1. Notably, the standard deviation of the total and projected track length of the CORSIKA 8 simulation in homogeneous media is, compared to the other frameworks, increased by two orders of magnitude. This is due to an outlier in the simulation with a very small track length, caused by a photonuclear interaction whose secondary particles are currently not further propagated. Otherwise, the medians of the observables all agree on a $10 \%$ level. 


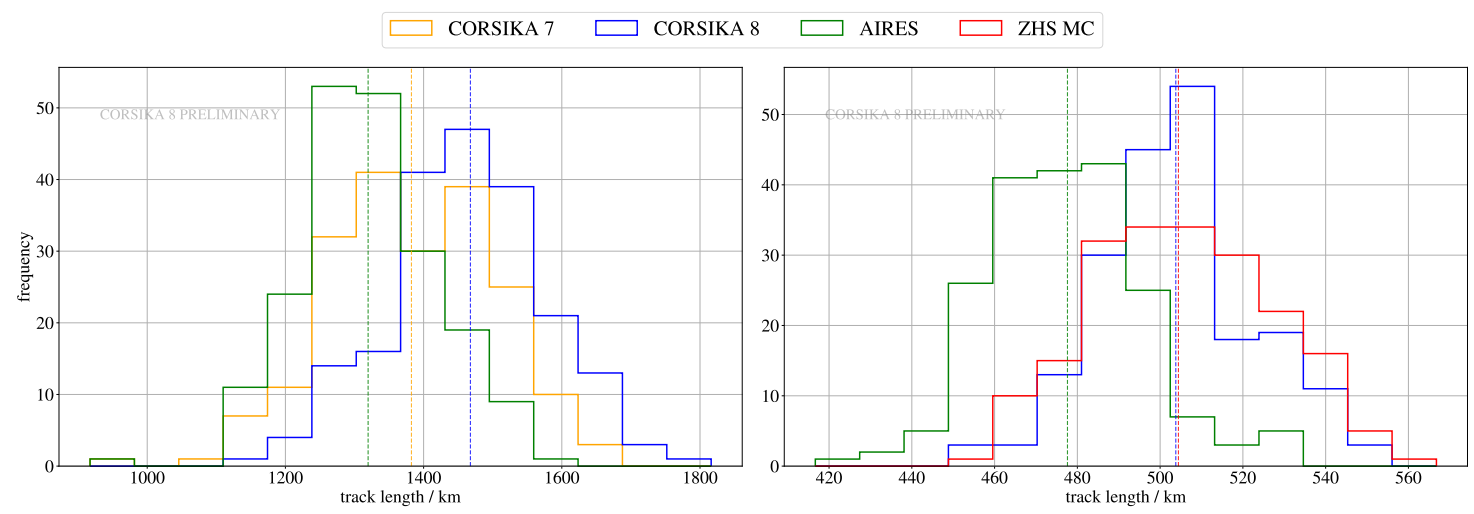

Figure 4: Distribution of the excess charged track length, i.e. the difference between the sum of all electron track lengths projected onto the shower axis and the sum of all positron track lengths projected onto the shower axis, for 200 showers initiated by $1 \mathrm{TeV}$ electrons. The left plot shows the distribution for an inhomogeneous atmosphere, the right plot for a homogeneous atmosphere. The dashed lines indicate the medians of the corresponding distributions.

Table 1: Track length medians for 200 showers, initiated by $1 \mathrm{TeV}$ electrons, for simulations in inhomogeneous and homogeneous atmospheres. The parentheses indicate the standard deviation.

\begin{tabular}{lrrrrrrrr}
\hline & \multicolumn{3}{c}{ inhomogeneous } & & \multicolumn{3}{c}{ homogeneous } \\
\cline { 2 - 3 } \cline { 8 - 9 } & & C7 & C8 & AIRES & & C8 & AIRES & ZHS MC \\
\hline Total track / km & $8083(724)$ & $7868(642)$ & $8047(620)$ & & $2891(202)$ & $2916(3)$ & $3126(2)$ \\
Proj. track. / km & $7750(697)$ & $7837(640)$ & $7681(594)$ & & $2776(194)$ & $2771(3)$ & $2924(2)$ \\
Excess / km & $1483(129)$ & $1493(115)$ & $1427(106)$ & & $549(43)$ & $520(19)$ & $574(22)$ \\
Proj. excess / km & $1382(121)$ & $1468(113)$ & $1320(99)$ & & $504(40)$ & $478(19)$ & $504(22)$ \\
Proj. excess / Total & 0.171 & 0.187 & 0.164 & & 0.174 & 0.164 & 0.161 \\
\hline
\end{tabular}

\section{Outlook}

In this work, the results of the first simulations of electromagnetic showers within the framework CORSIKA 8, using PROPOSAL as an electromagnetic model, have been presented and compared to other shower simulation tools. Although these comparisons revealed several differences, the general results are promising.

Further work on both PROPOSAL and the interface to CORSIKA 8 is necessary in the future. In order to improve the longitudinal distribution, the photohadronic interaction of photons will be implemented. Furthermore, a correct treatment of the LPM effect in inhomogeneous media still needs to be applied. Both additions will be especially relevant for the simulation of higher-energetic showers.

With regard to the lateral distribution, the deflection of primary particles in stochastic interactions as well as the deflection of bremsstrahlung photons will be added in the near future. Including these effects may solve the deviations currently observed in the lateral profile of CORSIKA 8.

Parallel to these improvements, further comparisons to these and other simulation frameworks, 
as well as simulations in other media such as ice, are going to be necessary. Since CORSIKA 8 is still in an early development state, runtime comparisons to the other frameworks are not yet conclusive and need to be conducted in future analyses.

\section{Acknowledgments}

We acknowledge funding by the Deutsche Forschungsgemeinschaft under the grant numbers RH 25/9-1 (J. S., W. R.) and SA 3867/1-1 (A. S.). This work has been supported by the DFG, Collaborative Research Center SFB 876, project C3 (http://sfb876.tu-dortmund.de).

This work has received financial support from Xunta de Galicia (Centro singular de investigación de Galicia accreditation 2019-2022), by European Union ERDF, by the María de Maeztu Units of Excellence program MDM-2016-0692, the Spanish Research State Agency and from Ministerio de Ciencia e Innovación PID2019-105544GB-I00 and RED2018-102661-T (RENATA).

\section{References}

[1] D. Heck et al., CORSIKA: A Monte Carlo code to simulate extensive air showers, Tech. Rep. FZKA-6019, Forschungszentrum Karlsruhe, 1998.

[2] W. R. Nelson, H. Hirayama, and D. W. O. Rogers, The EGS4 code system, Tech. Rep. SLAC-265, Stanford Linear Accelerator Center, 1985.

[3] J.-H. Koehne et al., Comput. Phys. Commun. 184 (2013) 2070-2090.

[4] M. Dunsch et al., Comput. Phys. Commun. 242 (2019) 132-144.

[5] J.-M. Alameddine et al., J. Phys. Conf. Ser. 1690 (2020) 012021.

[6] S. J. Sciutto, AIRES - A system for air shower simulations, 2019.

[7] E. Zas, F. Halzen, and T. Stanev, Phys. Rev. D 45 (1992) 362-376.

[8] J. Alvarez-Muñiz, W. R. Carvalho, Jr., M. Tueros, and E. Zas, Astropart. Phys. 35 (2012) 287-299.

[9] H. Koch and J. Motz, Reviews of modern physics 31 (1959) 920.

[10] T. Stanev and C. Vankov, Comput. Phys. Commun. 16 (1979) 363-372.

[11] J. C. Butcher and H. Messel, Phys. Rev. 112 (1958) 2096-2106.

[12] J. C. Butcher and H. Messel, Nucl. Phys. 20 (1960) 15-128.

[13] B. Rossi and K. Greisen, Rev. Mod. Phys. 13 (1941) 240-309.

[14] Y.-S. Tsai, Rev. Mod. Phys. 46 (1974) 815.

[15] S. M. Seltzer and M. J. Berger, Int. J. Appl. Radiat. Isot. 33 (1982) 1219-1226.

[16] V. L. Highland, Nuclear Instruments and Methods 129 (1975) 497-499.

[17] M. Reininghaus and R. Ulrich, EPJ Web Conf. 210 (2019) 02011.

[18] J. Alvarez-Muñiz, E. Marqués, R. A. Vázquez, and E. Zas, Phys. Rev. D 74 (2006) 023007. 


\section{Full Authors List: CORSIKA 8 Collaboration}

Jean-Marco Alameddine ${ }^{b}$, Johannes Albrecht ${ }^{b}$, Jaime Alvarez-Muñiz ${ }^{r}$, Antonio Augusto Alves $\mathrm{Jr}^{d}$, Juan Ammerman-Yebra ${ }^{r}$ Luisa Arrabito $^{a}$, Dominik Baack ${ }^{b}$, Konrad Bernlöhr ${ }^{c}$, Marcus Bleicher ${ }^{o}$, Johan Bregeon ${ }^{s}$, Mathieu Carrere ${ }^{a}$, Hans Dembinski ${ }^{b}$, Hannah Elfner $^{i}$, Dominik Elsässer ${ }^{b}$, Ralph Engel ${ }^{d}$, Fan Hu${ }^{p}$, Anatoli Fedynitch ${ }^{j}$, Dieter Heck ${ }^{d}$, Tim Huege ${ }^{d, e}$, Karl-Heinz Kampert ${ }^{k}$, Nikolaos Karastathis ${ }^{d}$, Lukas Nellen ${ }^{f}$, Maximilian Nöthe ${ }^{b}$, David Parello ${ }^{t}$, Tanguy Pierog ${ }^{d}$, Maria Pokrandt ${ }^{d}$, Anton Poctarev ${ }^{d}$, Remy Prechelt ${ }^{l}$, Maximilian Reininghaus ${ }^{d, m}$, Wolfgang Rhode ${ }^{b}$, Felix Riehn ${ }^{h, r}$, Maximilian Sackel ${ }^{b}$, Alexander Sandrock ${ }^{u}$, Pranav Sampathkumar ${ }^{d}$, Michael Schmelling ${ }^{c}$, André Schmidt ${ }^{d}$, Günter Sigl ${ }^{n}$, Jan Soedingrekso ${ }^{b}$, Bernhard Spaan ${ }^{b}$, Donglian Xu ${ }^{q}$ and Enrique Zas ${ }^{r}$

${ }^{a}$ Laboratoire Univers et Particules, Université de Montpellier 2, Montpellier, France, ${ }^{b}$ Experimentelle Physik 5, TU Dortmund, Dortmund, Germany, ${ }^{c}$ Max Planck Institute for Nuclear Physics, Heidelberg, Germany, ${ }^{d}$ Institute for Astroparticle Physics, Karlsruhe Institute of Technology, Karlsruhe, Germany, ${ }^{e}$ Astrophysical Institute, Vrije Universiteit Brussel, Brussels, Belgium ${ }^{f}$ National Autonomous University of Mexico, Mexico City, Mexico, ${ }^{g}$ Instituto de Tecnologías en Detección y Astropartículas, Buenos Aires, Argentina, ${ }^{h}$ Laboratory of Instrumentation and Experimental Particles, Lisbon, Portugal, ${ }^{i}$ Helmholtzzentrum für Schwerionenforschung, Darmstadt, Germany, ${ }^{j}$ Institute for Cosmic Ray Research, The University of Tokyo, Tokyo, Japan, ${ }^{k}$ Lehrstuhl für Astroteilchenphysik, Bergische Universität Wuppertal, Wuppertal, Germany, ${ }^{l}$ Department of Physics \& Astronomy, University of Hawai'i at Manoa, Honolulu, USA, ${ }^{m}$ Instituto de Tecnologías en Detección y Astropartículas, Buenos Aires, Argentina, ${ }^{n}$ II Institut für Theoretische Physik, Universität Hamburg, Hamburg, Germany, ${ }^{o}$ Johann-Wolfgang-Goethe-Universitaet, Frankfurt am Main, Germany, ${ }^{p}$ Peking University, Beijing, China, ${ }^{q}$ Tsung-Dao Lee Institute, Shanghai, China, ${ }^{r}$ Instituto Galego de Física de Altas Enerxías, Universidade de Santiago de Compostela, Santiago de Compostela, Spain, ${ }^{s}$ Laboratoire de Physique Subatomique et de Cosmologie, Grenoble, France, ${ }^{t}$ LIRMM, Univ Montpellier, CNRS, Montpellier, France, ${ }^{u}$ National Research Nuclear University, Moscow Engineering Physics Institute, Moscow, Russia. 